\title{
Do biomarkers trump behavior?
}

\section{To the editor:}

The article entitled "Biomarkers trump behavior in mental illness diagnosis"1 seems to misinterpret the growing data on biomarkers in psychiatry in a dangerous way. Research on biomarkers is undoubtedly important, particularly for the insights it provides on pathophysiology and for its potential relevance in determining prognosis and response to treatment. But suggesting that biomarkers "trump behavior" in psychiatric diagnosis seems inappropriate, at least in the current state of medical knowledge.

First, most studies dealing with biomarkers in psychiatric disorders, including the one mentioned in your article ${ }^{2}$, include patients diagnosed by current psychiatric classifications such as the DSM-IV, which are based on symptoms and clinical history. So, behavior is actually the gold standard in these studies, and stating that a biomarker is accurate based on these data merely means that it correlates well with symptoms. There is no way, therefore, that biomarkers could possibly trump behavior in such studies; on the contrary, if clinical symptoms are as dubious and misguiding for diagnosis as your piece seems to suggest, the markers that have been shown to correlate with them will also be so.

Second, and perhaps more important, the assumption that psychiatric diagnoses as we know them today actually represent individual pathophysiologic entities is risky. As current definitions of psychiatric disorders are based on symptoms, the belief that a specific disorder corresponds to a specific neurobiological alteration is a mere assumption ${ }^{3}$, and one can easily make the case that one disorder may have many pathophysiologic bases or, conversely, that similar pathophysiologic processes can lead to different disorders in different people. Therefore, using biomarkers, genetic studies or any kind of objective biological data for psychiatric diagnosis would first require a remodeling of psychiatric nosology, which might lead to the spectrum of mental disorders being very different from what it is today.

The points made above are not meant to invalidate the work on biomarkers, which can be valuable in many ways, such as in understanding the pathophysiology of mental disorders, predicting prognosis and response to treatment, and possibly helping early detection of illness in high-risk patients. Markers trumping behavior in psychiatric diagnosis, however, will only make sense when our understanding of the neurobiological bases of mental illness reaches a level that allows us to rewrite the science of psychiatry. This seems to be decades away, and one might argue that it may not happen at all. Until that day comes, advocating the use of markers in diagnosis is likely to end up doing more harm than good, leading clinicians more and more toward the easy solution of classifying people into disorders instead of listening to them and trying to understand their suffering.

\section{Olavo B Amaral}

Departamento de Bioquímica, Instituto de Ciências Básicas da Saúde, Universidade Federal do Rio Grande do Sul, Rua Ramiro Barcelos, 2600 (Prédio Anexo), CEP 90035-003

Porto Alegre, Rio Grande do Sul, Brazil.

e-mail:olavoamaral@yahoo.com.br

\section{COMPETING INTERESTS STATEMENT}

The author declares that he has no competing financial interests.

1. Haag, A.L. Nat. Med. 13, 3 (2007).

2. Huang, J.T. et al. PLoS Med. 3, e428 (2006)

3. Kendler, K.S. Am. J. Psychiatry 163, 1138-1146 (2006).

\section{Reply to 'Alice Dautry' profile}

To the editor:

We were dismayed to read the article on the new directorship at the Institut Pasteur and the steps that are being taken to pacify the campus after a recent period of upheaval ${ }^{1}$.

As researchers working at Pasteur, we welcome the attention the media has paid to this institution and are of course happy with the favorable light cast on the present director, Alice Dautry. Her job is indeed a very difficult one and the benefits of a sympathetic press are appreciated.

Most researchers at Pasteur support her in her endeavors, and everyone is committed to seeing this centenary institution attain even higher levels of excellence. However, we cannot let pass without protest the unfair, inelegant and inappropriate portrait of Philippe Kourilsky painted in this article. Kourilsky served as the institute's director for six years and his dedication to the institute is beyond doubt. He always had the best interests of the institute in mind.

It was under Kourilsky that many of the measures intended to boost competition, including the idea of promoting young scientists through the creation of five-year programs, were initiated. Dautry herself maintained support for many of those initiatives, an indication of how important they are.

Certain remarks quoted in the article deny Kourilsky the respect he deserves. What's more, they convey the impression that everyone found the Institut Pasteur an unpleasant place to work. There are alternative views of this period in the institute's recent history. Although some people obviously have unpleasant memories of that time, Nature Medicine's readers have the right to know that that opinion is by no means unanimous.

Some of the signatories of this letter were appointed by Kourilsky, but were also either confirmed or chosen for new positions by Dautry.

António Bandeira, Françoise Barré-Sinoussi, Pierre-André Cazenave, Stewart Cole, Ana Cumano, James Di Santo, Nicole Guiso, Alain Israël, Pablo Pereira \& Paulo Vieira

Institut Pasteur, 25 Rue du Docteur Roux, 75015 Paris, France.

e-mail:pvieira@pasteur.fr

\section{COMPETING INTERESTS STATEMENT}

The authors declare that they have no competing financial interests.

1. Butler, D. Nat. Med. 12, 1230 (2006) 